\title{
On the Duality between Existence and Information
}

\author{
David Ellerman \\ Philosophy Department, \\ University of California at Riverside
}

\section{Contents}

1 Introduction 2

I The Mathematics of Information as Distinctions 4

2 The Elements-Distinctions Duality 4

3 Partitions and Equivalence Relations 5

4 The Lattice of Partitions $\quad 6$

5 The Logic of Partitions $\quad 8$

6 Logical information theory $\quad 9$

7 Objective Indefiniteness and Quantum Mechanics $\quad 11$

8 The two dual conceptions of reality 12

II The Existence-Information Duality 12

9 Analogies to illustrate objective indefiniteness $\quad 12$

9.1 Getting from A to B . . . . . . . . . . . . . . . . . . . . . . 12

9.2 The two ways to interpret a superposition using an isosceles triangle . . . . . . . . 13

9.3 An example using Guy Fawkes masks . . . . . . . . . . . . . . . . . . . . . . . 15

9.4 Projecting a superposition vector to the eigen-axes . . . . . . . . . . . . . 15

10 Existence-Information Duality Illustrated by the Two Lattices 16

10.1 Moving up in the two lattices . . . . . . . . . . . . . . . . . . . . . . . . . . . . . . . . .

10.2 The two creation stories . . . . . . . . . . . . . . . . . . . . . 17

10.3 Reality consists of indefinite states as well as definite eigenstates . . . . . . . . . . 18

10.4 Moving vertically or moving horizontally in the two lattices . . . . . . . . . . . . 18

11 Conclusions $r$

\footnotetext{
Abstract

Recent developments in pure mathematics (category theory) and in mathematical logic (partition logic) have uncovered a fundamental duality between "existence" and "information." In
} 
logic, the duality is between the Boolean logic of subsets (mis-specified as the logic of "propositions") and the logic of quotient sets, equivalence relations, or partitions. The analogue to an element of a subset is the notion of a distinction (a pair of elements in different blocks) of a partition, and that leads to a whole stream of dualities or analogies-including the development of new logical foundations for information theory parallel to Boole's development of logical finite probability theory.

After outlining these dual concepts in mathematical terms, we turn to a more metaphysical speculation about two dual notions of reality, a fully definite notion using Boolean logic and appropriate for classical physics, and the other objectively indefinite notion using partition logic which turns out to be appropriate for quantum mechanics. The existence-information duality is used to intuitively illustrate these two dual notions of reality. The elucidation of the objectively indefinite notion of reality leads to the "killer application" of the existence-information duality, namely the interpretation of quantum mechanics.

\section{Introduction}

Recent developments in mathematics (category theory) and in mathematical logic (the logic of partitions) have revealed a duality, which involves a whole set of analogies, between the basic notions of existence and information.

One way to trace these developments is to go back to the beginning of what is today called "propositional" logic. It was originally developed as the logic of subsets, not the logic of propositions.

The algebra of logic has its beginning in 1847, in the publications of Boole and De Morgan. This concerned itself at first with an algebra or calculus of classes, to which a similar algebra of relations was later added. Though it was foreshadowed in Boole's treatment of "Secondary Propositions," a true propositional calculus perhaps first appeared from this point of view in the work of Hugh MacColl, beginning in 1877. [4, 155-56]

In the original setting of the logic of subsets, the variables stood for subsets of some universe set $U$ and the "connectives" were subset operations (e.g., "or" was the union of subsets and "and" was the intersection of subsets). A valid formula or tautology was a formula so that for any universe $U$ (with one or more elements), and for any subsets of $U$ substituted for the variables in the formula, the formula would evaluate to $U$. Boole himself realized that for the determination of valid formulas, it suffices to take $U$ as just a one element set with subsets that could be represented by $1=U$ and $0=\emptyset$. That a one-element universe suffices is a theorem of subset logic, not a definition. Thus it would also suffice to take the variables as standing for propositions with $1=T$ (true) and $0=F$ (false). The theorem in the Boolean logic of subsets that for validity it suffices to take $U=1$ has morphed into the definition of validity as truth-table validity (see any logic textbook today). What is missed by focusing on the special case of propositional logic instead of the Boolean logic of subsets? To answer this, we need to look at a recent development in pure mathematics

The theory of categories was formalized in 1945 [12] and it provided a precise notion of duality (essentially "turn around the arrows"). Boole's notion of a subset of a universe set $U$ is then seen to have a dual in the notion of a quotient set of $U$. The notion of a quotient set is equivalent to the notions of a equivalence relation on $U$ or a partition on $U$. More generally in other categories than the category of sets, the notion of a suboject or "part" is dual to the notion of a quotient object. "The dual notion (obtained by reversing the arrows) of 'part' is the notion of partition." [20, p. 85]

It is this duality that is "missed" by focusing on the special case of "propositional" logic rather than full subset logic. Propositions don't have a dual; subsets do. When the idea of the logic of subsets is combined with the category-theoretic dual concept of a partition or equivalence relation, then the idea arises of a dual logic of partitions or equivalence relations. It has been known since Dedekind's work on lattices that the lattice operations of join and meet could be defined for partitions 
or equivalence relations (e.g., the lattice of partitions on a set gives the standard example of a nondistributive lattice). But no other logical operations were defined even though Gian-Carlo Rota and colleagues wrote on the "logic" of certain types of equivalence relations [13]. In a 2001 memorial volume for Rota, it was observed:

Equivalence relations are so ubiquitous in everyday life that we often forget about their proactive existence. Much is still unknown about equivalence relations. Were this situation remedied, the theory of equivalence relations could initiate a chain reaction generating new insights and discoveries in many fields dependent upon it.

This paper springs from a simple acknowledgement: the only operations on the family of equivalence relations fully studied, understood and deployed are the binary join $\vee$ and meet $\wedge$ operations. [3, p. 445]

Since Rota was essentially the author's mentor in mathematics, after his premature death I undertook to develop the general logic of partitions. ${ }^{1}$ And this did "initiate a chain reaction generating new insights and discoveries in many fields" as expected.

The first chain-reaction result from the development of partition logic was obtained by imitating Boole's next step of developing finite logical probability theory as the normalized counting measure of the elements in subsets-which is why "Probabilities" are mentioned in the title of Boole's book on the laws of thought [2]. In the duality between subsets and partitions, it is easily seen that the analogue to an element of a subset is a "distinction" of a partition. A partition $\pi=\left\{B, B^{\prime}, \ldots\right\}$ on a set $U$ is set of pair-wise disjoint subsets $B, B^{\prime}, \ldots$ of $U$ (called the blocks of the partition) whose union is $U$, and a distinction of $\pi$ is an ordered pair $\left(u, u^{\prime}\right)$ of elements $u, u^{\prime} \in U$ in different blocks of the partition $\pi$. Hence the next step was to imitate Boole by defining the theory of the normalized counting measure of the distinctions of a partition. It became clear that this provides new foundations for information theory based on partition logic, and that the normalized counting measure of the distinctions was a logical notion of entropy or information content, the logical entropy $h(\pi)$ of a partition $\pi([6] ;[8])$.

The idea that information is based on distinctions or differences is quite old. In James Gleick's book, The Information: A History, A Theory, A Flood, he noted the focus on distinctions or differences in the seventeenth century polymath, John Wilkins, who was a founder of the Royal Society. In 1641, the year before Newton was born, Wilkins published one of the earliest books on cryptography, Mercury or the Secret and Swift Messenger, which not only pointed out the fundamental role of differences but noted that any (finite) set of different things could be encoded by words in a binary code.

For in the general we must note, That whatever is capable of a competent Difference, perceptible to any Sense, may be a sufficient Means whereby to express the Cogitations. It is more convenient, indeed, that these Differences should be of as great Variety as the Letters of the Alphabet; but it is sufficient if they be but twofold, because Two alone may, with somewhat more Labour and Time, be well enough contrived to express all the rest. [33, Chap. XVII, p. 69]

Wilkins explains that a five letter binary code would be sufficient to code the letters of the alphabet since $2^{5}=32$.

Thus any two Letters or Numbers, suppose A.B. being transposed through five Places, will yield Thirty Two Differences, and so consequently will superabundantly serve for the Four and twenty Letters... .[33, Chap. XVII, p. 69]

\footnotetext{
${ }^{1}$ See [7] for the development starting with basic concepts up to the correctness and completeness proofs for a tableau system of partition logic, and see [9] for a more introductory treatment.
} 
As Gleick noted:

Any difference meant a binary choice. Any binary choice began the expressing of cogitations. Here, in this arcane and anonymous treatise of 1641, the essential idea of information theory poked to the surface of human thought, saw its shadow, and disappeared again for [three] hundred years. [14, p. 161]

Gleick is here dating the development of modern information theory from Claude Shannon's monumental work published in 1948 ([24]; see also [25]). This is how the development of the logic of partitions, dual to the Boolean logic of subsets and using the analogy between the elements-of-asubset and the distinctions-of-a-partition, reveals a duality between logical probability theory and logical information theory.

Another chain-reaction result at the level of meta-physics, inspired by the notion of information as distinctions, was a different notion of reality as involving objective indefiniteness-which in turn provided a way to give a realistic interpretation of quantum mechanics ([10]; [11]).

In sum, these developments can be seen all as aspects of the basic duality between existence and information.

This paper has a mathematical part and a philosophical part. There is so much philosophical "blah-blah" written about "information" that the first part of the paper outlines the mathematical basis for treating information as being based on distinctions. Then the second part of the paper will indulge in more philosophical speculations about the duality between existence and informationwhere the "killer application" is the interpretation of quantum mechanics.

\section{Part I}

\section{The Mathematics of Information as Distinctions}

\section{The Elements-Distinctions Duality}

If partitions are dual to subsets, then what is the dual concept that corresponds to the notion of elements of a subset? The notion dual to the elements of a subset is the notion of the distinctions of a partition (pairs of elements in distinct blocks of the partition). The duality between elements of a subset and distinctions of a partition already appears in the very notion of a function between sets. What binary relations, i.e., subsets $R \subseteq X \times Y$, specify functions $f: X \rightarrow Y$ ?

A binary relation $R \subseteq X \times Y$ transmits elements if for each element $x \in X$, there is an ordered pair $(x, y) \in R$ for some $y \in Y$.

A binary relation $R \subseteq X \times Y$ reflects elements if for each element $y \in Y$, there is an ordered pair $(x, y) \in R$ for some $x \in X$.

A binary relation $R \subseteq X \times Y$ transmits distinctions if for any pairs $(x, y)$ and $\left(x^{\prime}, y^{\prime}\right)$ in $R$, if $x \neq x^{\prime}$, then $y \neq y^{\prime}$.

A binary relation $R \subseteq X \times Y$ reflects distinctions if for any pairs $(x, y)$ and $\left(x^{\prime}, y^{\prime}\right)$ in $R$, if $y \neq y^{\prime}$, then $x \neq x^{\prime}$.

The dual role of elements and distinctions can be seen if we translate the usual characterization of the binary relations that define functions into the elements-and-distinctions language. A binary relation $R \subseteq X \times Y$ defines a function $X \rightarrow Y$ if it is defined everywhere on $X$ and is single-valued. But "being defined everywhere" is the same as transmitting elements, and being single-valued is the same as reflecting distinctions:

a binary relation $R$ is functional if it transmits elements and reflects distinctions. 
What about the other two special types of relations, i.e., those which transmit distinctions or reflect elements? The two important special types of functions are the injections and surjections, and they are defined by the other two notions:

a functional relation is injective if it transmits distinctions, and a functional relation is surjective if it reflects elements.

Given a functional relation or set function $f: X \rightarrow Y$ with domain $X$ and codomain $Y$, the subset-partition duality follows. A subset of the codomain is determined as the image $f(X)$ of an injective function $f$, and a partition on the domain is determined as the coimage (or inverse-image) $\left\{f^{-1}(y)\right\}_{y \in Y}$ of a surjective function $f$.

These elements-and-distinctions definitions of injections and surjections yield "arrow-theoretic" characterizations which can then be lifted into any category to provide the usual category-theoretic dual definitions of monomorphisms (injections for set functions) and epimorphisms (surjections for set functions). Two set functions $f, g: X \rightarrow Y$ are different, i.e., $f \neq g$, if there is an element $x$ of $X$ such that their values $f(x)$ and $g(x)$ are a distinction of $Y$, i.e., $f(x) \neq g(x)$. Hence if $f$ and $g$ are followed by a function $h: Y \rightarrow Z$, then the compositions $h f, h g: X \rightarrow Y \rightarrow Z$ must be different if $h$ preserves distinctions (so that the distinction $f(x) \neq g(x)$ is preserved as $h f(x) \neq h g(x)$ ), i.e., if $h$ is injective. In the category of sets, $h$ being injective is characterized by " $h f=h g$ implies $f=g$ " which is the general category-theoretic definition of a monomorphism.

In a similar manner, if we had preceded $f$ and $g$ where $f \neq g$ by a function $h: W \rightarrow X$, then the compositions $f h, g h: W \rightarrow X \rightarrow Y$ must be different if $h$ reflects elements (so that the element $x$ where $f$ and $g$ differ is sure to be in the image of $h$ ), i.e., if $h$ is surjective. In the category of sets, $h$ being surjective is characterized by " $f h=g h$ implies $f=g$ " which is the general category-theoretic definition of an epimorphism.

Hence the dual interplay of the notions of elements and distinctions can be seen as yielding the arrow-theoretic characterizations of injections and surjections which are lifted into the general categorical definitions of monomorphisms and epimorphisms, and which, in part, motivate the reverse-the-arrows duality of category theory.

\section{Partitions and Equivalence Relations}

Partitions are often considered in the guise of equivalence relations so it will be useful to first establish some terminology. An equivalence relation is a binary relation $E \subseteq U \times U$ that is reflexive, symmetric, and transitive. Every equivalence relation on a set $U$ determines a partition on $U$ where the equivalence classes are the mutually exclusive and jointly exhaustive blocks of the partition. Conversely, every partition on a set determines an equivalence relation on the set (two elements are equivalent if they are in the same block of the partition). The notions of a partition on a set and an equivalence relation on a set are thus interdefinable. Indeed, equivalence relations and partitions are often considered as the "same" as in the conventional practice (N.B. not used here) of defining the "lattice of partitions" as the lattice of equivalence relations.

For the purposes of partition logic, it is important to consider the complementary type of binary relation. A partition relation $R \subseteq U \times U$ is irreflexive (i.e., $(u, u) \notin R$ for any $u \in U$ ), symmetric [i.e., $\left(u, u^{\prime}\right) \in R$ implies $\left(u^{\prime}, u\right) \in R$ ], and anti-transitive in the sense that if $\left(u, u^{\prime}\right) \in R$, then for any $a \in U$, either $(u, a) \in R$ or $\left(a, u^{\prime}\right) \in R$ [i.e., $U \times U-R=R^{c}$ is transitive]. Thus as binary relations, equivalence relations and partition relations are complementary. That is, $E \subseteq U \times U$ is an equivalence relation if and only if (iff) $U \times U-E=E^{c} \subseteq U \times U$ is a partition relation. A partition relation is also called an apartness relation in computer science. Any partition relation is the set of distinctions of a partition and vice-versa.

There is a natural ("built-in") closure operation on $U \times U=U^{2}$. The closure operation is "built-in" to $U$ in the sense that no topology, ordering relations, or other structure is assumed on 
$U$. A subset $C \subseteq U^{2}$ is closed if it contains the diagonal $\Delta=\{(u, u) \mid u \in U\}$ (i.e., is reflexive), if $\left(u, u^{\prime}\right) \in C$ implies $\left(u^{\prime}, u\right) \in C$ (i.e., is symmetric), and if $\left(u, u^{\prime}\right)$ and $\left(u^{\prime}, u^{\prime \prime}\right)$ are in $C$, then $\left(u, u^{\prime \prime}\right)$ is in $C$ (i.e., is transitive). Thus the closed sets of $U^{2}$ are precisely the equivalence relations on $U$. The intersection of any number of closed sets is closed. Given a subset $S \subseteq U^{2}$, the closure $\bar{S}$ is the reflexive, symmetric, and transitive closure of $S$. The formation of the closure $\bar{S}$ can be divided into two steps. First $S^{*}$ is formed from $S$ by adding any diagonal pairs $(u, u)$ not already in $S$ and by symmetrizing $S$, i.e., adding $\left(u^{\prime}, u\right)$ if $\left(u, u^{\prime}\right) \in S$. To form the transitive closure of $S^{*}$, for any finite sequence $u=u_{1}, u_{2}, \ldots, u_{n}=u^{\prime}$ with $\left(u_{i}, u_{i+1}\right) \in S^{*}$ for $i=1, \ldots, n-1$, add $\left(u, u^{\prime}\right)$ and $\left(u^{\prime}, u\right)$ to the closure. The result is the reflexive, symmetric, and transitive closure $\bar{S}$ of $S$. The complements of the closed sets in $U \times U$ are defined as the open sets. Let $\mathcal{O}(U \times U)$ be the set of open subsets of $U \times U$, the partition relations on $U$. As usual, the interior $\operatorname{int}(S)$ of any subset $S \subseteq U \times U$ is defined as the complement of the closure of its complement: $\operatorname{int}(S)=\left({\overline{S^{c}}}^{c}\right.$.

It should, however, be carefully noted that the "closure space" $U \times U$ is not a topological space, i.e., the closure operation on $U^{2}$ is not a topological closure operation in the sense that the union of two closed set is not necessarily closed (or, equivalently, the intersection of two open sets is not necessarily open).

\section{The Lattice of Partitions}

Just as the usual treatment of the Boolean lattice of all subsets of a universe $U$ assumes that $U$ has one or more elements, so our treatment of the lattice of all partitions on $U$ will assume that $U$ has two or more elements. This avoids the "degenerate" special cases of there being only one subset of an empty $U$ and only one partition on a singleton $U$.

A pair $\left(u, u^{\prime}\right) \in U \times U$ is a distinction or dit (from DIsTinction) of the partition $\pi$ if there are distinct blocks $B, B^{\prime} \in \pi$ with $u \in B$ and $u^{\prime} \in B^{\prime}$. The set of distinctions of a partition $\pi$, its dit set denoted $\operatorname{dit}(\pi) \subseteq U \times U$, is the partition seen as a partition relation:

$$
\operatorname{dit}(\pi)=\bigcup_{B, B^{\prime} \in \pi, B \neq B^{\prime}} B \times B^{\prime}
$$

(where it is understood that the union includes both the Cartesian products $B \times B^{\prime}$ and $B^{\prime} \times B$ for $\left.B \neq B^{\prime}\right)$.

A pair $\left(u, u^{\prime}\right) \in U \times U$ is an indistinction or indit (from INDIsTinction) of a partition $\pi$ if $u$ and $u^{\prime}$ belong to the same block of $\pi$. The set of indistinctions of a partition $\pi$, its indit set denoted $\operatorname{indit}(\pi)=U \times U-\operatorname{dit}(\pi)$, is the complementary equivalence relation:

$$
\operatorname{indit}(\pi)=\bigcup_{B \in \pi} B \times B=U \times U-\operatorname{dit}(\pi)=\operatorname{dit}(\pi)^{c} .
$$

In terms of the closure space structure on $U \times U$, the open sets (partition relations), denoted $\mathcal{O}(U \times U)$, are the dit sets $\operatorname{dit}(\pi)$ of partitions, and the complementary closed sets (equivalence relations) are the indit sets indit $(\pi)$ of partitions.

When equivalence relations and partitions were considered as the "same," then the "lattice of partitions," e.g., Birkhoff [1] or Grätzer [15], was traditionally defined as isomorphic to the lattice of equivalence relations where the partial order was inclusion between the equivalence relations as subsets of $U \times U$. But since equivalence relations and partition relations are complementary subsets of the closure space $U \times U$, we have two anti-isomorphic lattices with opposite partial orders.

Which lattice should be used in partition logic? Since the Boolean lattice of subsets uses sets of elements with the partial order as inclusion between sets of elements, the dual logic should use the lattice of partition relations, which are sets of distinctions, with the partial order as inclusion between the sets of distinctions. 
The lattice of partitions $\Pi(U)$ on $U$ can be defined using the usual definition of a partition. The equivalent definitions in terms of the partition relations which are the open subsets $\mathcal{O}(U \times U)$ of the closure space (i.e., dit sets).

The partial order in the lattice is the refinement relation: given two partitions $\pi=\{B\}_{B \in \pi}$ and $\sigma=\{C\}_{C \in \sigma}$,

$\sigma \preceq \pi$ (read " $\pi$ refines $\sigma$ " or " $\sigma$ is refined by $\pi$ ") if for any block $B \in \pi$, there is a block $C \in \sigma$ with $B \subseteq C .^{2}$

The equivalent definition using dit sets (i.e., partition relations) is just inclusion:

$$
\sigma \preceq \pi \operatorname{iff} \operatorname{dit}(\sigma) \subseteq \operatorname{dit}(\pi) .
$$

The lattice of partitions $\Pi(U)$ is the partition analogue of the powerset Boolean lattice $\wp(U)$. In the powerset lattice, the partial order is inclusion of elements, and in the partition lattice, it is inclusion of distinctions. The top of the powerset Boolean lattice $\wp(U)$ is the universe set $U$ (all elements) and the bottom is the null set $\emptyset$ (no elements). The top of the partition lattice $\Pi(U)$ is the discrete partition $\mathbf{1}=\left\{\{u\},\left\{u^{\prime}\right\}, \ldots\right\}$ where each block is a singleton (all possible distinctions), and the bottom is the indiscrete partition (or blob) $\mathbf{0}=\{\{U\}\}$ with only one block consisting of all of $U$ (no distinctions).

The join $\pi \vee \sigma$ is the partition whose blocks are the non-empty intersections $B \cap C$ of the blocks of the two partitions. The join in the powerset Boolean algebra is given by the union of the subsets of elements and the equivalent dit-set definition in $\mathcal{O}(U \times U)$ is simply the union of the sets of distinctions: $\operatorname{dit}(\pi \vee \sigma)=\operatorname{dit}(\pi) \cup \operatorname{dit}(\sigma)$.

Recall that the closure operator on the closure space was not topological in the sense that the union of two closed sets is not necessarily closed and thus the intersection of two open sets (i.e., two dit sets) is not necessarily open. Hence the definition of the meet of two partitions requires some more complication. In $\mathcal{O}(U \times U)$, the dit set of the meet of two partitions is the interior of the intersection of the two dit sets, i.e.,

$$
\operatorname{dit}(\sigma \wedge \pi)=\operatorname{int}(\operatorname{dit}(\sigma) \cap \operatorname{dit}(\pi)) .
$$

That completes the definition of the lattice of partitions $\Pi(U)$ and its representation as the lattice $\mathcal{O}(U \times U)$ of open subsets of the product $U \times U$ :

$$
\Pi(U) \cong \mathcal{O}(U \times U) .
$$

Representation of the lattice of partitions $\Pi(U)$

as the lattice of open subsets $\mathcal{O}(U \times U)$

For $U=\{a, b, c\}$, the Boolean lattice of subsets and the partition lattice can be illustrated as follows.

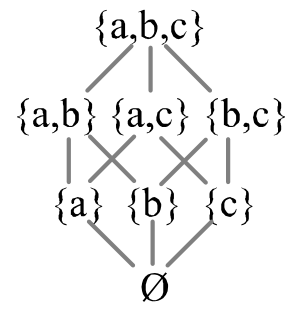

Subset lattice

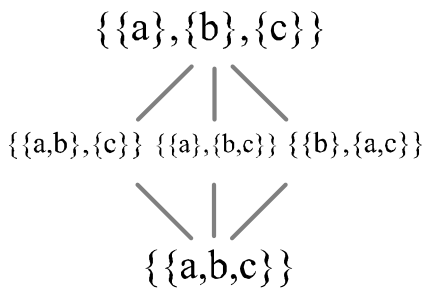

Partition lattice

Figure 1: Lattices of subsets and of partitions

\footnotetext{
${ }^{2}$ Note that the opposite partial order is called the "refinement" ordering in the customary "upside down" treatment of the lattice of partitions. Gian-Carlo Rota used to joke that it should be called the "unrefinement" relation. Indeed, in a recent book on Rota-style combinatorial theory, that relation is sensibly called "reverse refinement" [19, p. 30].
} 
The analogies between the lattice of subsets $\wp(U)$ and the lattice of partitions $\Pi(U)$ are summarized in the following table.

\begin{tabular}{|l|l|l|}
\cline { 2 - 3 } Analogies & Boolean lattice of subsets & Lattice of partitions \\
\hline \hline "Elements" & Elements of subsets & Distinctions of partitions \\
\hline Partial order & Inclusion of elements & Inclusion of distinctions \\
\hline Join & $\begin{array}{l}\text { Elements of join are } \\
\text { union of elements }\end{array}$ & $\begin{array}{l}\text { Distinctions of join are } \\
\text { union of distinctions }\end{array}$ \\
\hline Meet & $\begin{array}{l}\text { Largest subset } \\
\text { of only common elements }\end{array}$ & $\begin{array}{l}\text { Largest partition relation } \\
\text { of only common distinctions }\end{array}$ \\
\hline Top & Subset $U$ with all elements & Partition 1 with all distinctions \\
\hline Bottom & Subset $\emptyset$ with no elements & Partition 0 with no distinctions \\
\hline \multicolumn{3}{|c|}{ Table 1: Elements-distinctions analogies between } \\
the Boolean lattice of subsets and the lattice of partitions.
\end{tabular}

\section{The Logic of Partitions}

Now that we have represented a partition $\pi$ as a certain type of subset, i.e., an open subset or partition relation $\operatorname{dit}(\pi) \subseteq U \times U$, we can just follow the analogies with the logic of subsets to generate the logic of partitions. It was noted that previously only the partition operations of join and meet had been defined and studied. But now we just "follow the analogy" to have a general algorithm to translate the logical operations of the Boolean logic of subsets into logical operations on partitions. For any binary logical subset operation, symbolized by \#, apply that set operation to the dit sets of two partitions $\pi$ and $\sigma$ to arrive at $\operatorname{dit}(\pi) \# \operatorname{dit}(\sigma) \subseteq U \times U$. Since that subset of $U \times U$ may not be an open subset, we apply the interior operation to obtain the open subset $\operatorname{int}[\operatorname{dit}(\pi) \# \operatorname{dit}(\sigma)]$ which is then defined as the dit set $\operatorname{dit}(\pi \# \sigma)$ of the partition $\pi \# \sigma$.

For instance, the first logical operation on partitions that one would want to define beyond join and meet (where this algorithm gives the results defined above) is the implication operation $\sigma \Rightarrow \pi$ on partitions. Since the subset operation for the implication or conditional $A \Rightarrow B$ for $A, B \subseteq U$ is $A^{c} \cup B$, the dit set $\operatorname{dit}(\sigma \Rightarrow \pi)$ would be defined as: $\operatorname{int}\left[\operatorname{dit}(\sigma)^{c} \cup \operatorname{dit}(\pi)\right]$.

While that is the partition implication operation used in the logic of partitions, there is an alternative characterization of the implication operation that is more useful. Given $\pi=\{B\}$ and $\sigma=\{C\}$, the partition $\sigma \Rightarrow \pi$ has the same blocks $B$ as $\pi$ except when there is a block $C \in \sigma$ such that $B \subseteq C$ and then $B$ is "discretized", i.e., replaced by the singletons $\{u\}$ for $u \in B$. If we think of the whole $B$ as a mini-blob $\mathbf{0}_{B}$ and the discretized $B$ as a mini discrete partition $\mathbf{1}_{B}$, then the implication $\sigma \Rightarrow \pi$ is just the characteristic or indicator function that applied to $B \in \pi$ returns $\mathbf{1}_{B}$ if there is a $C \in \sigma$ with $B \subseteq C$ and otherwise returns $\mathbf{0}_{B}$.

The first check on the implication operation is that it captures the partial ordering relation. For instance, in the Boolean lattice of subsets, $A \Rightarrow B=U$ (top of the lattice) is equivalent to the inclusion partial order $A \subseteq B$. Analogously for partitions, when $\sigma \Rightarrow \pi=\mathbf{1}$ (top of the lattice of partitions) that means all the blocks $B \in \pi$ were discretized, i.e., for every $B \in \pi$ there was a $C \in \sigma$ such that $B \subseteq C$, which is the definition of the refinement partial order $\sigma \preceq \pi$.

The same result can be obtained using the dit-set definition of the implication. No dit set contains any self-pairs $(u, u) \in \Delta$ of the diagonal. The dit set of the discrete partition $\mathbf{1}$ is the set $U \times U-\Delta$ of all possible distinctions. If $\operatorname{dit}(\sigma \Rightarrow \pi)=\operatorname{int}\left[\operatorname{dit}(\sigma)^{c} \cup \operatorname{dit}(\pi)\right]=\operatorname{dit}(\mathbf{1})$ then $U \times U-\Delta \subseteq \operatorname{dit}(\sigma)^{c} \cup \operatorname{dit}(\sigma)$ where $\operatorname{dit}(\sigma)^{c}=\operatorname{indit}(\sigma)$ as an equivalence relation must contain $\Delta$ (since all equivalence relations are reflexive), so $\operatorname{dit}(\sigma)^{c} \cup \operatorname{dit}(\pi)=U \times U$ and thus $\operatorname{dit}(\sigma) \subseteq \operatorname{dit}(\pi)$ which is equivalent to $\sigma \preceq \pi$.

Thus we may define the logical operations on partitions using the analogy with the logical operations on subsets so we can develop any logical formulas such as $(\sigma \Rightarrow \pi) \vee((\sigma \Rightarrow \pi) \Rightarrow \pi)$ where 
the variables stand for partitions on $U$ rather than subsets of $U$. And we define a valid formula of partition logic, i.e., a partition tautology, analogously as a formula such that for any universe $U$ (two or more elements to avoid the degeneracy of $\mathbf{0}=\mathbf{1}$ ) and for any partitions on $U$ substituted for the variables, the formula evaluates to the discrete partition $\mathbf{1}$ (the top of the algebra of partitions). For instance, it is easily checked that the formula $(\sigma \Rightarrow \pi) \vee((\sigma \Rightarrow \pi) \Rightarrow \pi)$ (the partition version of the weak law of excluded middle for the $\pi$-negation $\stackrel{\pi}{\neg} \sigma=\sigma \Rightarrow \pi)$ is a partition tautology since every block $B \in \pi$ is either discretized in $\sigma \Rightarrow \pi$ or, if not, then it has to be discretized in $(\sigma \Rightarrow \pi) \Rightarrow \pi$.

It is also easy to show that all partition tautologies are subset tautologies. There are only two partitions $\mathbf{1}$ and $\mathbf{0}$ on a two-element set 2 and the partition operations on those two partitions have the same "truth table" as the subset operations on the two subsets $\emptyset$ and 1 for a one-element universe $U=1$. Thus if a partition formula always evaluates to $\mathbf{1}$ for any $U$ then it does so for the two-element universe 2 and thus the same subset formula always evaluates to 1 in its truth table which suffices to establish that the formula is a subset tautology. The converse is not true as is easily checked for the law of excluded middle $\sigma \vee(\sigma \Rightarrow \mathbf{0})$ which evaluates to $\sigma$ for any $\sigma \neq \mathbf{0}$. The valid formulas of partition logic are neither contained in nor contain the valid formulas of intuitionistic propositional logic.

More of the analogies between subset logic and partition logic are summarized in the following table.

\begin{tabular}{|c||c|c|}
\hline Analogies & Subset logic & Partition logic \\
\hline \hline 'Elements' & Elements $u$ of $S$ & Dits $\left(u, u^{\prime}\right)$ of $\pi$ \\
\hline Order & Inclusion $S \subseteq T$ & Refinement: $\operatorname{dit}(\sigma) \subseteq \operatorname{dit}(\pi)$ \\
\hline Top of order & $U$ all elements & $\operatorname{dit}(\mathbf{1})=U^{2}-\Delta$, all dits \\
\hline Bottom of order & $\emptyset$ no elements & $\operatorname{dit}(\mathbf{0})=\emptyset$, no dits \\
\hline Variables in formulas & Subsets $S$ of $U$ & Partitions $\pi$ on $U$ \\
\hline Operations & Subset ops. & Partition ops. \\
\hline Formula $\Phi(x, y, \ldots)$ holds & $u$ element of $\Phi(S, T, \ldots)$ & $\left(u, u^{\prime}\right) \operatorname{dit}$ of $\Phi(\pi, \sigma, \ldots)$ \\
\hline Valid formula & $\Phi(S, T, \ldots)=U, \forall S, T, \ldots$ & $\Phi(\pi, \sigma, \ldots)=\mathbf{1}, \forall \pi, \sigma, \ldots$ \\
\hline
\end{tabular}

Table 2: Analogies between subset logic and partition logic.

\section{Logical information theory}

For any finite set $X$, a (finite) measure $\mu$ is a function $\mu: \wp(X) \rightarrow \mathbb{R}$ such that:

1. $\mu(\emptyset)=0$,

2. for any $E \subseteq X, \mu(E) \geq 0$, and

3. for any disjoint subsets $E_{1}$ and $E_{2}, \mu\left(E_{1} \cup E_{2}\right)=\mu\left(E_{1}\right)+\mu\left(E_{2}\right)$.

Any finite set $X$ has the counting measure ||$: \wp(X) \rightarrow \mathbb{R}$ and normalized counting measure $\frac{\mid l}{|X|}: \wp(X) \rightarrow \mathbb{R}$ defined on the subsets of $X$. Hence for finite $U$, we have the counting measure || and the normalized counting measure $\frac{||}{|U \times U|}$ defined on $\wp(U \times U)$. Boole used the normalized counting measure $\frac{1 \mid}{|U|}$ defined on the power-set Boolean algebra $\wp(U)$ to define the logical probability $\operatorname{Pr}(S)=\frac{|S|}{|U|}$ of an event $S \subseteq U$.[2] In view of the analogy between elements ("its") in subset logic and dits in partition logic, the construction analogous to the logical probability is the normalized counting measure applied to dit sets. That is the definition of the:

$$
h(\pi)=\frac{|\operatorname{dit}(\pi)|}{|U \times U|}
$$

Logical entropy of a partition $\pi$. 
In a random (i.e., equiprobable) drawing of an element from $U$, the event $S$ occurs with the probability $\operatorname{Pr}(S)$. If we take two independent (i.e., with replacement) random drawings from $U$, i.e., pick a random ordered pair from $U \times U$, then $h(\pi)$ is the probability that the pair is a distinction of $\pi$, i.e., that $\pi$ distinguishes-just as $\operatorname{Pr}(S)$ is the probability that a random drawing from $U$ yields an element of $S$.

These analogies are summarized in the following table which uses the language of probability theory (e.g., set of outcomes, events, the occurrence of an event):

\begin{tabular}{|c||c|c|}
\hline Analogies & Subset logic & Partition logic \\
\hline \hline 'Outcomes' & Elements $u$ of $S$ & Ordered pairs $\left(u, u^{\prime}\right) \in U \times U$ \\
\hline 'Events' & Subsets $S$ of $U$ & Partitions $\pi$ of $U$ \\
\hline 'Event occurs' & $u \in S$ & $\left(u, u^{\prime}\right) \in \operatorname{dit}(\pi)$ \\
\hline Norm. counting measure & $\operatorname{Pr}(S)=\frac{|S|}{|U|}$ & $h(\pi)=\frac{|\operatorname{dit}(\pi)|}{|U \times U|}$ \\
\hline Interpretation & Prob. event $S$ occurs & Prob. partition $\pi \operatorname{distinguishes}$ \\
\hline
\end{tabular}

Table 3: Analogies between logical probability theory and logical information theory.

Thus logical entropy $h(\pi)$ is the simple quantitative measure of the distinctions of a partition $\pi$ just as the logical probability $\operatorname{Pr}(S)$ is the quantitative measure of the elements in a subset $S$. In short, information theory is to partition logic as probability theory is to ordinary subset logic.

To generalize logical entropy from partitions to finite probability distributions, note that:

$$
\operatorname{dit}(\pi)=\left\{B \times B^{\prime}: B, B^{\prime} \in \pi, B \neq B^{\prime}\right\}=U \times U-\{B \times B: B \in \pi\} .
$$

Using $p_{B}=\frac{|B|}{|U|}$, we have:

$$
h(\pi)=\frac{|\operatorname{dit}(\pi)|}{|U \times U|}=\frac{|U|^{2}-\sum_{B \in \pi}|B|^{2}}{|U|^{2}}=1-\sum_{B \in \pi}\left(\frac{|B|}{|U|}\right)^{2}=1-\sum_{B \in \pi} p_{B}^{2} .
$$

Recall that an ordered pair $\left(u, u^{\prime}\right) \in B \times B$ for some $B \in \pi$ is an indistinction or indit of $\pi$ where $\operatorname{indit}(\pi)=U \times U-\operatorname{dit}(\pi)$. Hence in a random drawing of a pair from $U \times U, \sum_{B \in \pi} p_{B}^{2}$ is the probability of drawing an indistinction, while $h(\pi)=1-\sum_{B \in \pi} p_{B}^{2}$ is the probability of drawing a distinction.

Entropies will be defined both for partitions on finite sets and for finite probability distributions (i.e., finite random variables). Given a random variable $u$ with the probability distribution $p=$ $\left(p_{1}, \ldots, p_{n}\right)$ over the $n$ distinct values $U=\left\{u_{1}, \ldots, u_{n}\right\}$, a distinction of the discrete partition on $U$ is just a pair $\left(u_{i}, u_{j}\right)$ with $i \neq j$ and with the probability $p_{i} p_{j}$. Applying the previous notion to the logical entropy of a partition to this case with $p_{B}=p_{i}$ (where $B=\left\{u_{i}\right\}$ ), we have the:

$$
\begin{gathered}
h(p)=\sum_{i} p_{i}\left(1-p_{i}\right)=1-\sum_{i} p_{i}^{2} \\
\text { Logical entropy of a finite probability distribution } p .
\end{gathered}
$$

The notion of logical entropy finally provides a logical foundation for information theory based directly on the notion of distinctions. The concepts and results of Shannon's information theory can be redeveloped in logical information theory ([6]; [8]). Shannon's notion of entropy $H(\pi)=$ $\sum_{B \in \pi} p_{B} \log _{2}\left(1 / p_{B}\right)=-\sum_{B \in \pi} p_{B} \log _{2}\left(p_{B}\right)$ is not a measure (in the mathematical sense given above), only has a direct interpretation in special cases (i.e., the number of equal binary partitions it takes to differentiate $2^{n}$ entities), and was applied in what Shannon always called "theory of communications" ([24]; [25]).

Both Shannon entropy and logical entropy generalize directly to quantum information theory where the probability distribution $p$ is replaced by a density matrix $\rho$ and the summation is replaced by the trace of a matrix: 


$$
\begin{gathered}
\text { Shannon entropy: } H(p)=-\sum_{i} p_{i} \log _{2}\left(p_{i}\right) \text { becomes } H(\rho)=-\operatorname{tr}\left[\rho \log _{2} \rho\right] \text {, } \\
\text { Logical entropy: } h(p)=1-\sum_{i} p_{i}^{2} \text { becomes } h(\rho)=1=\operatorname{tr}\left[\rho^{2}\right] .
\end{gathered}
$$

The quantum information theory version of Shannon entropy is usually called Von Neumann entropy [22]. Here again, the notion of quantum logical entropy provides a simpler and clearer "logical" treatment of information and hence some quantum information theorists ([30]; [31]) are redeveloping the concepts using quantum logical entropy instead of Von Neumann entropy.

We find this framework of partitions and distinction most suitable (at least conceptually) for describing the problems of quantum state discrimination, quantum cryptography and in general, for discussing quantum channel capacity. In these problems, we are basically interested in a distance measure between such sets of states, and this is exactly the kind of knowledge provided by logical entropy [reference to [6]]. [30, p. 1]

Thus in both the classical and quantum context, the notion of information as distinctions is a developed mathematical theory.

\section{Objective Indefiniteness and Quantum Mechanics}

There has long been the notion of subjective or epistemic indefiniteness ("cloud of ignorance") that is slowly cleared up with more discrimination and distinctions (as in the game of Twenty Questions). But the conception of reality that seems appropriate for quantum mechanics is objective or ontological indefiniteness. The notion of objective indefiniteness in quantum mechanics (QM) has been most emphasized by the late Abner Shimony ([26], [27], [28]).

From these two basic ideas alone - indefiniteness and the superposition principle - it should be clear already that quantum mechanics conflicts sharply with common sense. If the quantum state of a system is a complete description of the system, then a quantity that has an indefinite value in that quantum state is objectively indefinite; its value is not merely unknown by the scientist who seeks to describe the system. [26, p. 47]

The fact that in any pure quantum state there are physical quantities that are not assigned sharp values will then mean that there is objective indefiniteness of these quantities. [28, p. 27]

Shimony also suggested that this interpretation of QM could be called the "Literal" interpretation.

These statements ... may collectively be called "the Literal Interpretation" of quantum mechanics. This is the interpretation resulting from taking the formalism of quantum mechanics literally, as giving a representation of physical properties themselves, rather than of human knowledge of them, and by taking this representation to be complete.[29, pp. 6-7]

The view that a description of a superposition quantum state is a complete description means that the indefiniteness of a superposition state is objective or ontological and not just subjective or epistemological.

Today, the idea that a quantum state is, in some sense, indefinite, blurred, or like a cloud is now rather commonplace even in the popular literature. Much of the literature on the interpretation of QM represents attempts to escape the standard Dirac-Von-Neumann QM in various flights of fancy (many worlds, hidden variables, etc.), while the literal or objective indefiniteness interpretation considered here is based on trying to directly make sense out a superposition as a complete description of an objectively indefinite state. 


\section{The two dual conceptions of reality}

There are two quite different notions of reality:

1. Definite (classical): the common-sense conception of objectively definite ("all the way down") reality assumed in classical physics and described logically in the Boolean logic of subsets where each element is either in a subset or its complement and that characterizes the element (principle of identity of indiscernibles), and

2. Indefinite (quantum): the conception of an objectively indefinite reality (with some definite "eigen-states") suggested by quantum physics and mathematically described by partitions.

Our common-sense conception of reality is one of definiteness, so where did this second idea come from? Was it an ad hoc idea pulled out of the air just to explain QM? The second conception comes out of mathematics itself. The two conceptions are based on the notion of duality in category theory which gives the duality between subsets and quotient sets (or equivalence relations or partitions).

What is the mathematical way to describe indefiniteness? Partitions! The basic idea is simple; start with what is taken as full definiteness and then factor or quotient out the "surplus" definiteness using an equivalence relation or partition.

Starting with some universe set $U$ of fully distinct and definite elements, a partition $\pi=\left\{B_{i}\right\}$ (i.e., a set of disjoint blocks $B_{i}$ that sum to $U$ ) collects together in a block (or cell) $B_{i}$ the distinct elements $u \in U$ whose distinctness is to be ignored or factored out, but the blocks are still distinct from each other. Each block represents the elements that are the same in some aspect (since each block is an equivalence class in some equivalence relation on $U$ ), so the block is indefinite between the elements within it. But different blocks are still distinct from each other in that aspect (i.e., are different equivalence classes).

\section{Part II}

\section{The Existence-Information Duality}

\section{Analogies to illustrate objective indefiniteness}

\subsection{Getting from $\mathrm{A}$ to $\mathrm{B}$}

In subset logic, each element of the universe set $U$ either definitely has or does not have a given property $P$ (represented as a subset of the universe). Moreover an element has properties "all the way down" so that two numerically distinct entities must differ by some property as in Leibniz's principle of the identity of indiscernibles. Change takes place by the definite properties changing.

In the logic of partitions, a partition $\pi=\left\{B_{i}\right\}$ is made up of disjoint blocks $B_{i}$ whose union is the universe set $U$ (the blocks are also thought of as the equivalence classes in an equivalence relation). The blocks in a partition have been distinguished from each other, but the elements within each block have not been distinguished from each other by that partition. Hence each block can be viewed as the set-theoretic version of a superposition of the distinct elements in the block. When more distinctions are made (the set-version of a measurement), the blocks get smaller and the partitions (set-version of mixed states) become more refined until the discrete partition $\mathbf{1}=\{\{u\}:\{u\} \subseteq U\}$ is reached where each block is a singleton (the set-version of a non-degenerate measurement). Change takes place by some attributes becoming more definite and other (incompatible) attributes becoming less definite.

For a "classical" hound to go from point $A$ to point $B$, there must be some trajectory of definite ground locations from $A$ to $B$. 
For a "quantum" hawk to go from point $A$ to point $B$, it would go from a definite perch at $A$ into a flight of indefinite ground locations, and then would have a definite perch again at $B .^{3}$

\begin{tabular}{|l|l|}
\hline $\begin{array}{l}\text { Classical trajectory from A to B. How a } \\
\text { hound goes from A to B. }\end{array}$ & $\begin{array}{l}\text { Subjective indefiniteness about classical } \\
\text { position ("cloud of ignorance"). }\end{array}$ \\
\hline $\begin{array}{l}\text { Objective indefiniteness of quantum } \\
\text { trajectory: definite position at A, } \\
\text { indefinite position in transition, and } \\
\text { definite position at B. How a hawk goes } \\
\text { from A to B. }\end{array}$
\end{tabular}

Figure 2: How a "classical" hound and a "quantum" hawk get from A to B.

The imagery of having a sharp focus versus being out of focus could also be used if one is clear that it is the reality itself that is in-focus or out-of-focus, not just the image through, say, a microscope. A classical trajectory is like a moving picture of sharp or definite in-focus realities, whereas the quantum trajectory starts with a sharply focused reality, goes out of focus, and then returns to an in-focus reality (by a "measurement").

\subsection{The two ways to interpret a superposition using an isosceles triangle}

Consider a simple example of an isosceles triangle with three angles $a, b$, and $c$ with the respective opposite sides $A, B$, and $C$ where the two angles $b$ and $c$ as well as their opposite sides $B$ and $C$ are equal. Each complete labelling represents a fully definite state.
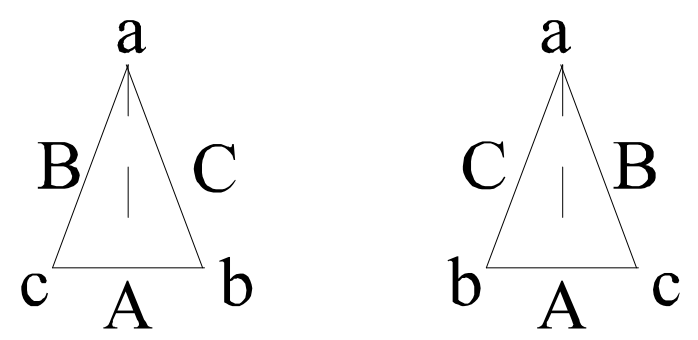

Figure 3: Two fully labelled isosceles triangles

But to represent the idea of an isosceles triangle, the labelling of the equal angles and equal sides is surplus information. The two definite states can be superposed or added together in an equivalence class (of states equated in the symmetry operation of reflection around the vertical dashed line) as a way to represent of objectively indefinite isosceles triangle prior to the distinctions introduced by the $b, c$ and $B, C$ labels.

\footnotetext{
${ }^{3}$ The "flights and perchings" metaphor is from William James [17, p. 158] and according to Max Jammer, that description "was one of the major factors which influenced, wittingly or unwittingly, Bohr's formation of new conceptions in physics." [18, p. 178] The hawks and hounds pairing comes from Shakespeare's Sonnet 91.
} 

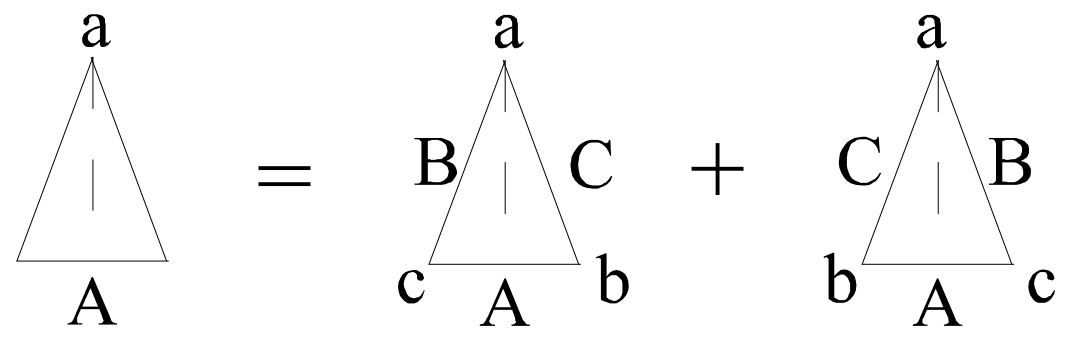

Figure 4: Objectively indefinite state as the superposition of fully definite eigenstates.

The objectively indefinite isosceles triangle (without the labels on the equal angles or equal sides) is analogous to the objectively indefinite states of quantum mechanics represented by the superposition of fully definite eigenstates. The difference between the indefinite and the definite triangles is not in the changing of any "substance" (changed angles or sides) but only the addition of information in the form of distinctions provided by the labels. The forcing of the making of a distinction is called a "measurement" in quantum mechanics.

There are always two ways to interpret a subset $S \subseteq U$ corresponding to the definite versus indefinite notions of reality.

1. A subset $S \in \wp(U)$ in a Boolean algebra may be classically seen as the extension of some property $P$ common to all and only the entities $u \in S$. Those entities all share the property $P$ but differ in some other properties.

2. The other "quantum" way to interpret a subset is as a block $S \in \pi$ in a partition where it can be taken as a single objectively indefinite entity that is indefinite between all the definite eigenstates $u \in S$.

These two ways to interpret a subset can be extended to the two ways to interpret a superposition such as the "addition" of the two fully-labelled isosceles triangles.

1. The definite classical way to interpret a superposition is like a double-exposure photograph (a relic of the film camera era) which is a superposition of two definite images. The standard imagery is the superposition of two definite classical waves.

2. The indefinite quantum way to interpret a superposition is as a single indefinite image that is definite in all the aspects common to the superposed entities but indefinite between the aspects where they differ.

The following diagram illustrates these two ways to interpret a superposition in the case of the isosceles triangle.

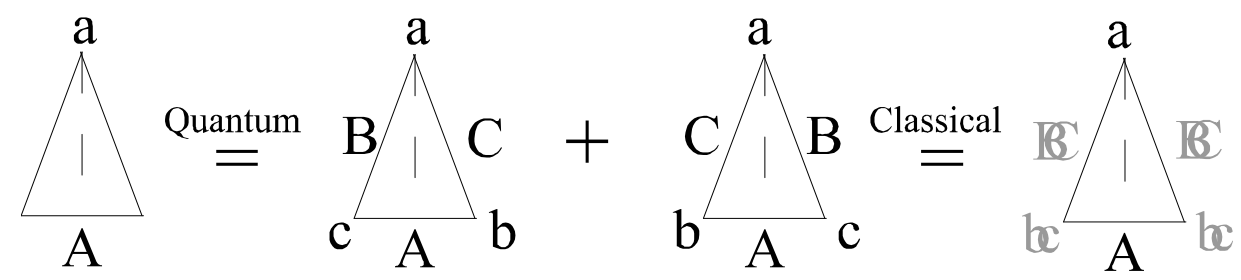

Figure 5: The indefinite quantum way and the definite classical way to interpret a superposition. 
If the two definite triangles in the superposition evolved over time (e.g., became $50 \%$ smaller), then the classical and quantum renditions of the superposition would evolve accordingly (e.g., become $50 \%$ smaller). The mathematics of linear superposition includes destructive or constructive interference and that would be reflected accordingly in both the classical interpretation of the superposition (like the usual interference of waves) and in the indefinite quantum interpretation of the superposition.

This latter point about the evolution of the indefinite version of a superposition is particularly difficult to understand (and perhaps should be marked with Bourbaki's "dangerous bend" symbol). But it follows from the indefinite interpretation of a "static" superposition as being definite on the aspects common to the superposed states and indefinite between the aspects where they differ. As the fully definite states in the superposition evolve, those aspects where they have in common or differ may change, and the indefinite version of the superposition will change accordingly. Thus an indefinite state can evolve, showing the effects of interference, without using the double-exposure classical interpretation of interference (e.g., the classical way to interpret the mathematics of waves or in Fourier analysis). ${ }^{4}$ The mathematics of superposition is the same, but there are the two ways to interpret it-in the classical or quantum ways.

This simple example suffices to indicate how the theme of partitions, distinctions, and information connects to the basic problem of interpreting quantum mechanics.

\subsection{An example using Guy Fawkes masks}

Guy Fawkes masks might also be used to intuitively illustrate the difference between the indefinite ("quantum") and the definite ("double exposure" or "classical") way to interpret a superposition. Suppose for the sake of the illustration that there are two eigenstates, mustache or goatee where a mask can have one or the other but not both. Mask 1 and Mask 2 represent the two eigenstates of "goatee" and "mustache" respectively, and the superposition "goatee + mustache" would under the indefinite "quantum" way of interpretation represent the Mask 3 that has neither of those distinctions, while the definite "classical" way of interpreting the superposition would be the Mask 4 with both.

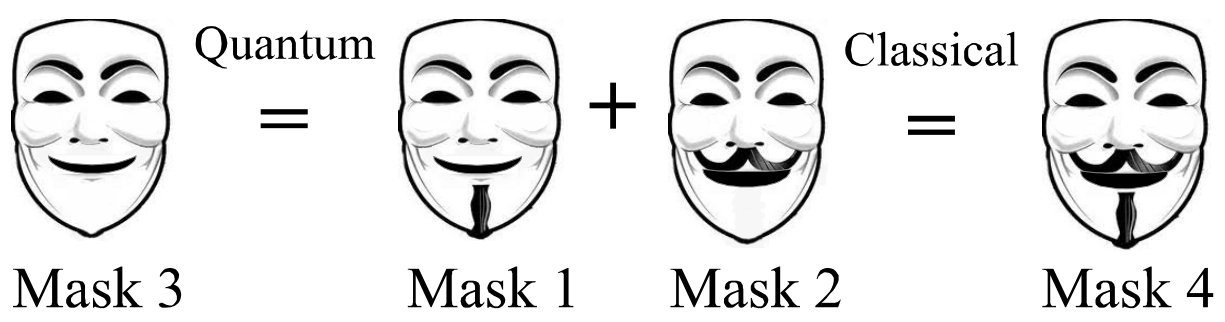

Figure 6: Guy Fawkes masks illustrating two ways to understand superposition $\mid$ goatee $\rangle+\mid$ mustache $\rangle$

\subsection{Projecting a superposition vector to the eigen-axes}

Consider a partition $\pi=\{\{b\},\{a, c\}\}$ on the three-element universe $U=\{a, b, c\}$. The block $S=$ $\{a, c\}$ is objectively indefinite between $\{a\}$ and $\{c\}$. This objective indefiniteness of $\{a, c\}$ is not welldescribed as saying that indefinite pre-distinction entity is "simultaneously both $\{a\}$ and $\{c\}$ " (like the common misdescription of the undetected particle "going through both slits" in the double-slit experiment). It is a misdescription like saying that the $45^{\circ}$ superposition vector $(1,0)+(0,1)$ on the real $x, y$-plane is simultaneously on the $x$-axis [as $(1,0)$ is] and on the $y$-axis [as $(0,1)$ is].

\footnotetext{
${ }^{4}$ See [11] or [10] for further mathematical analysis.
} 


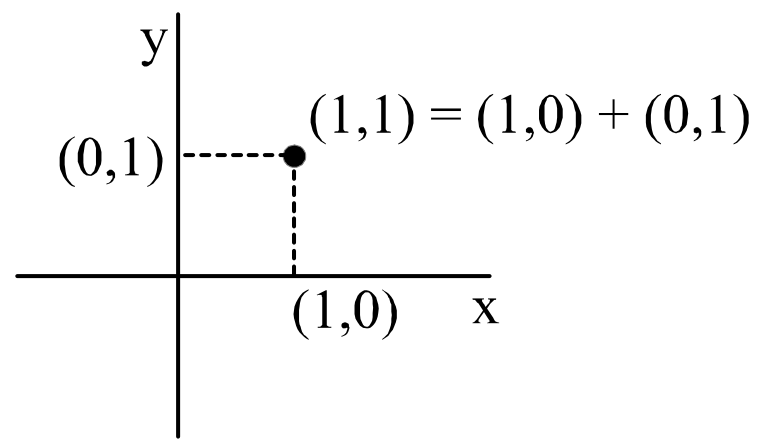

Figure 7: Superposition $(1,1)$ is not simultaneously $(1,0)$ and $(0,1)$.

A superposition of two sharp eigen-alternatives should not be thought of in quantum mechanics like a double-exposure photograph which has two fully definite images (e.g., simultaneously a picture of say $\{a\}$ and $\{c\}$ ). Instead of a double-exposure photograph, the superposition should be thought of as representing or describing one indefinite reality that with further distinctions could sharpen to either of the sharp realities in the superposition (mathematically, the distinctions project the $45^{\circ}$ superposition vector to either the $x$ or $y$ axis). There must be some way to indicate which definite states could be obtained by making further distinctions (measurements) in an indefinite state, and that is why the blurred or cloud-like indefinite state is represented by mathematically superposing the definite possibilities.

In quantum mechanics, the definite way to interpret a superposition of eigenstates is not available since the eigenstates are, in general, mutually exclusive ("orthogonal"). The idea of a quantum superposition as an indefinite state has been missing the "back story" to make sense out that conception of reality. That back story is provided, in part, by the logic of partitions, equally fundamental from the mathematical viewpoint as the dual Boolean subset logic, and by the logical information theory built on top of partition logic which explicates information using distinctions.

\section{Existence-Information Duality Illustrated by the Two Lat- tices}

\subsection{Moving up in the two lattices}

The two dual subset and partition logics are modeled by the two lattices (or, with more operations, algebras) of subsets and of partitions. The conceptual duality between the lattice of subsets (the lattice part of the Boolean algebra of subsets of $U$ ) and the lattice of partitions could be described using the rather meta-physical notions of existence (elements of subsets or "its") versus information (distinctions of partitions or "dits") or, equivalently, substance ${ }^{5}$ versus form (as in in-form-ation). The two lattices also correlate with the two different conceptions of reality, the classical vision of fully definite objective reality and the quantum vision of an objectively indefinite reality (made more definite by making more distinctions).

For each lattice where $U=\{a, b, c\}$, start at the bottom and move towards the top.

\footnotetext{
${ }^{5}$ Heisenberg identifies "substance" with energy.

Energy is in fact the substance from which all elementary particles, all atoms and therefore all things are made, and energy is that which moves. Energy is a substance, since its total amount does not change, and the elementary particles can actually be made from this substance as is seen in many experiments on the creation of elementary particles. [16, p. 63]
} 


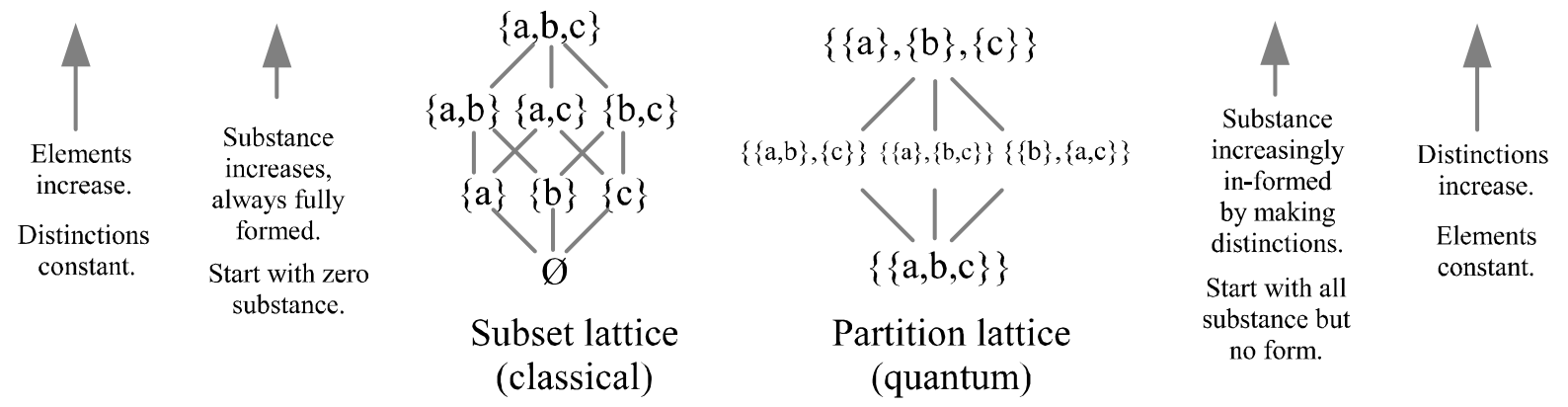

Figure 8: Moving up the subset and partition lattices.

At the bottom of the Boolean lattice is the empty set $\emptyset$ which represents no substance. As one moves up the lattice, new elements of substance always with fully definite properties are created until finally one reaches the top, the universe $U$. Thus new substance is created in moving up the lattice but each element is fully formed and distinguished in terms of its properties.

At the bottom of the partition lattice is the indiscrete partition or "blob" $\mathbf{0}=\{U\}$ (where the universe set $U$ makes one block) which represents all the substance but with no distinctions to in-form the substance. As one moves up the lattice, no new substance is created but distinctions are created that objectively in-form the indistinct elements as they become more and more distinct, until one finally reaches the top, the discrete partition $\mathbf{1}$, where all the eigen-elements of $U$ have been fully distinguished from each other. It was previously noted that a partition combines indefiniteness (within blocks) and definiteness (between blocks). At the top of the partition lattice, the discrete partition $\mathbf{1}=\{\{u\}:\{u\} \subseteq U\}$ is the result making all the distinctions to eliminate the indefiniteness. Thus one ends up at the "same" place (macro-universe of distinguished elements) either way, but by two totally different but dual ways. ${ }^{6}$

\subsection{The two creation stories}

The progress from bottom to top of the two lattices could also be described as two creation stories.

- Subset creation story: "In the Beginning was the Void", and then elements are created, fully propertied and distinguished from one another, until finally reaching all the elements of the universe set $U$.

- Partition creation story: "In the Beginning was the Blob", which is an undifferentiated perfectly symmetrical "substance," and then there is a "Big Bang" where the substance is being objectively in-formed (objectified information) by the making of distinctions (e.g., breaking symmetries $^{7}$ ) until the result is finally the singletons which designate the elements of the universe $U$.

These two creation stories might also be illustrated as follows.

\footnotetext{
${ }^{6}$ In treating the universe $U=\left\{u, u^{\prime}, \ldots\right\}$ and the discrete partition $\mathbf{1}=\left\{\{u\},\left\{u^{\prime}\right\}, \ldots\right\}$ as the "same" we are neglecting the distinction between $u$ and $\{u\}$ for $u \in U$.

${ }^{7}$ The breaking of a symmetry is one way to create information by making distinctions ([5]; [21]).
} 


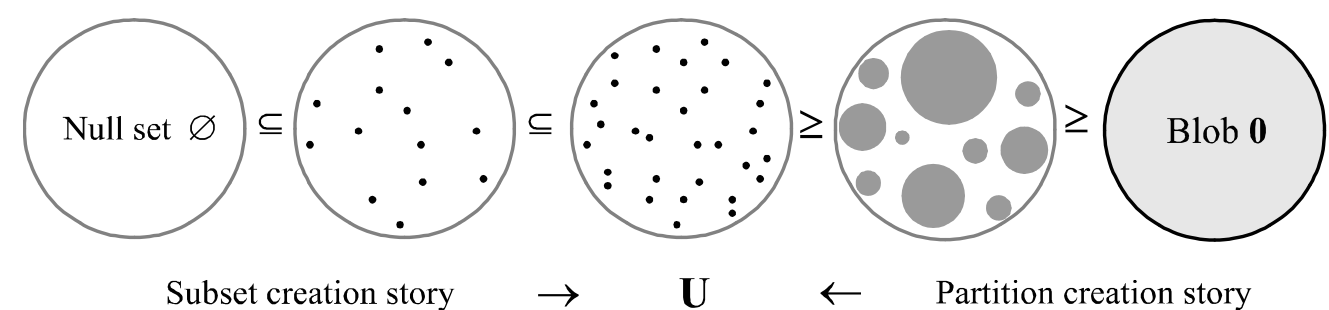

Figure 9: Creation as the creation of fully formed existence or the creation of objectified information.

One might think of the universe $U$ (in the middle of the above picture) as the macroscopic world of definite entities that we ordinarily experience. Common sense and classical physics assumes, as it were, the subset creation story on the left. But a priori, it could just as well have been the dual story, the partition creation story pictured on the right, which is a highly abstract way to describe the Big Bang theory of creation-constant substance (energy) in-formed by symmetry breaking. [23]

\subsection{Reality consists of indefinite states as well as definite eigenstates}

A set partition like $\{\{b\},\{a, c\}\}$ is a highly abstract analogue of what is called a "mixed state" in QM and a block like $\{a, c\}$ is the abstract analogue of what is called a "pure state" (in this case the superposition of the eigenstates $\{a\}$ and $\{c\}$ ). Under the objectively indefinite interpretation of a superposition state of a "particle," the particle is not simultaneously in the definite states $\{a\}$ and $\{c\}$, but is in the state of being indefinite between $\{a\}$ and $\{c\}$. The undetected particle that is often said to "go through both slits" in the double-slit experiment is actually in a spatially indefinite state $\mid$ slit 1$\rangle+\mid$ slit 2$\rangle$ which abstractly is like $\{a\}+\{c\}=\{a, c\}$. The common sense notion of space includes only the eigenstates of spatial position; it ignores the spatially indefinite states of a particle. That is why the question "which slit did the particle go through" has no "eigen-answer" since the undetected particle was not in an spatial eigenstate such as $\mid$ slit 1$\rangle$ or $\mid$ slit 2$\rangle$. In the abstract setting of the partition lattice, the answer to the "Where's Waldo" question of "where is the undetected particle" is the spatially indefinite state $\{a, c\}$ seen as $|s l i t 1\rangle+|s l i t 2\rangle$.

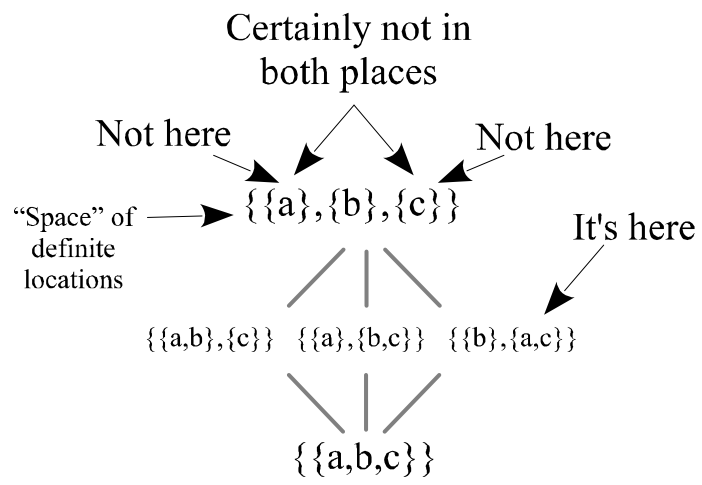

Partition lattice

Figure 10: Where's Waldo, the undetected particle in the double-slit experiment?

\subsection{Moving vertically or moving horizontally in the two lattices}

Returning to the two lattices, one might consider two types of processes within each lattice, the vertical movement described above and a "horizontal" sideways movement which we might refer to 
respectively as the "type 1 " and "type 2 " processes.

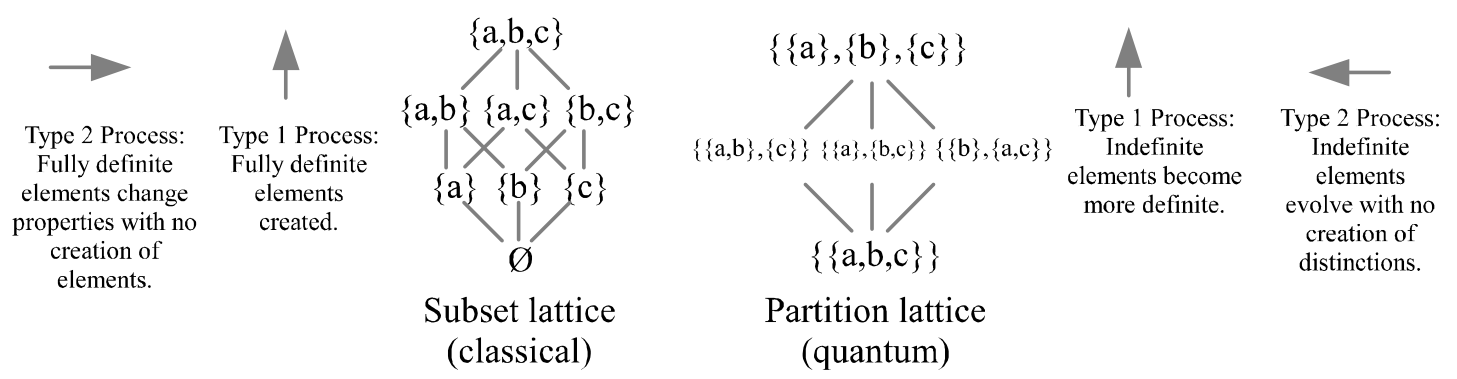

Figure 11: Type 1 and type 2 processes in the two lattices.

For the "classical" subset lattice, a horizontal type 2 process would go from one fully definite state to another, e.g., for a particle to go from one fully definite position and momentum to another as in a classical trajectory (in state space).

For the "quantum" partition lattice, a horizontal type 2 process would go from one indefinite state to another without creating or destroying any distinctions. In the mathematics of quantum mechanics, the degree of indistinctness or "overlap" between to quantum states $|\psi\rangle$ and $|\varphi\rangle$ is measured by their inner product $\langle\varphi \mid \psi\rangle$ so a type 2 process is one that preserves inner products (which is mathematically called a "unitary transformation" and in quantum mechanics is represented by the time dependent Schrödinger equation). In the quantum case, the moving-up type 1 process is one where distinctions are made so an objectively indefinite state becomes more definite-which is called a "measurement." These two processes, abstractly described in the partition lattice, correspond precisely to what Von Neumann called "type 1" and "type 2" processes in quantum mechanics [32, Chap. V, sec. 1].

\section{Conclusions}

The fundamental duality between existence and information appears in many forms. In category theory, it is the duality between subobjects and quotient objects which, in the case of sets, is the duality between subsets and partitions. The concept of a distinction of a partition is the correlate or analogue of the notion of an element of a subset. When what is today called "propositional" logic is respecified as the Boolean logic of subsets, then it can be seen that there is a dual logic of partitions. Moreover just as Boole developed logical probability as the normal counting measure on subsets (by counting elements), so the notion of logical entropy can be developed as the normalized counting measure on partitions (by counting distinctions)-which provides new logical foundations for information theory (in both the ordinary and quantum versions).

Turning more to metaphysical speculation, we have seen that the mathematically dual concepts are correlated with two dual notions of reality:

1. the common sense notion of a fully definite reality assumed in classical physics where the concepts of Boolean logic are appropriate, and

2. the notion of an objectively indefinite reality suggested by quantum mechanics where the concepts of partition logic are appropriate.

The second part of the paper focused on intuitively illustrating this notion of an objectively indefinite reality and connecting it to partitional concepts and to quantum mechanics. In this manner, the "killer application" of the development of the existence-information duality is the elucidation and interpretation of quantum mechanics. 


\section{References}

[1] Birkhoff, Garrett 1948. Lattice Theory. New York: American Mathematical Society.

[2] Boole, George 1854. An Investigation of the Laws of Thought on which are founded the Mathematical Theories of Logic and Probabilities. Cambridge: Macmillan and Co.

[3] Britz, Thomas, Matteo Mainetti and Luigi Pezzoli 2001. Some operations on the family of equivalence relations. In Algebraic Combinatorics and Computer Science: A Tribute to GianCarlo Rota. H. Crapo and D. Senato eds., Milano: Springer: 445-59.

[4] Church, Alonzo 1956. Introduction to Mathematical Logic. Princeton: Princeton University Press.

[5] Collier, John. 1996. Information Originates in Symmetry Breaking. Symmetry: Science and Culture. 7: 247-56.

[6] Ellerman, David 2009. Counting Distinctions: On the Conceptual Foundations of Shannon's Information Theory. Synthese. 168 (1 May): 119-149.

[7] Ellerman, David 2010. The Logic of Partitions: Introduction to the Dual of the Logic of Subsets. Review of Symbolic Logic. 3 (2 June): 287-350.

[8] Ellerman, David. 2013. An Introduction to Logical Entropy and Its Relation to Shannon Entropy. International Journal of Semantic Computing. 7 (2): 121-45.

[9] Ellerman, David. 2014. An Introduction of Partition Logic. Logic Journal of the IGPL. 22 (1): $94-125$.

[10] Ellerman, David. 2014. Partitions and Objective Indefiniteness in Quantum Mechanics. arXiv:1401.2421v2 [quant-ph].

[11] Ellerman, David. (forthcoming). On the Objective Indefiniteness Interpretation of Quantum Mechanics. In The Mammoth Book of Quantum Mechanics Interpretations, edited by Ulf Edvinsson. Open Academic Press.

[12] Eilenberg, S. and S. Mac Lane. 1945. General Theory of Natural Equivalences. Transactions of the American Mathematical Society. 58, No2, 231-94.

[13] Finberg, David, Matteo Mainetti and Gian-Carlo Rota 1996. The Logic of Commuting Equivalence Relations. In Logic and Algebra. Aldo Ursini and Paolo Agliano ed., New York: Marcel Dekker: 69-96.

[14] Gleick, James 2011. The Information: A History, A Theory, A Flood. New York: Pantheon.

[15] Grätzer, George 2003. General Lattice Theory (2nd ed.). Boston: Birkhäuser Verlag.

[16] Heisenberg, Werner 1958. Physics 83 Philosophy: The Revolution in Modern Science. New York: Harper Torchbooks.

[17] James, William 1952 (1890). The Principles of Psychology (Great Books Series \#53). Chicago: Encyclopedia Britannica.

[18] Jammer, Max 1966. The Conceptual Development of Quantum Mechanics. New York: McGrawHill.

[19] Kung, Joseph P.S., Gian-Carlo Rota and Catherine H. Yan 2009. Combinatorics: The Rota Way. New York: Cambridge University Press. 
[20] Lawvere, F. William and Robert Rosebrugh 2003. Sets for Mathematics. Cambridge: Cambridge University Press.

[21] Muller, Scott J. 2007. Asymmetry: The Foundation of Information. Berlin: Springer Verlag.

[22] Nielsen, M., and I. Chuang. 2000. Quantum Computation and Quantum Information. Cambridge: Cambridge University Press.

[23] Pagels, Heinz. 1985. Perfect Symmetry: The Search for the Beginning of Time. New York: Simon and Schuster.

[24] Shannon, Claude E. 1948. A Mathematical Theory of Communication. Bell System Technical Journal. 27: 379-423; 623-56.

[25] Shannon, Claude E. and Warren Weaver 1964. The Mathematical Theory of Communication. Urbana: University of Illinois Press.

[26] Shimony, Abner 1988. The reality of the quantum world. Scientific American. 258 (1): 46-53.

[27] Shimony, Abner 1989. Conceptual foundations of quantum mechanics. In The New Physics. Paul Davies ed., Cambridge: Cambridge University Press: 373-395.

[28] Shimony, Abner 1989. Search for a Worldview Which Can Accommodate Our Knowledge of Microphysics. In Philosophical Consequences of Quantum Theory: Reflections on Bell's Theorem. James T. Cushing and Ernan McMullin eds., Notre Dame, IN: University of Notre Dame Press: 25-37.

[29] Shimony, Abner. 1999. Philosophical and Experimental Perspectives on Quantum Physics. In Philosophical and Experimental Perspectives on Quantum Physics: Vienna Circle Institute Yearbook 7, Dordrecht: Springer Science+Business Media: 1-18.

[30] Tamir, Boaz, and Eliahu Cohen. 2015. Logical Entropy for Quantum States. http://de.arxiv.org/abs/1412.0616v2, January.

[31] Tamir, Boaz, and Eliahu Cohen. 2015. A Holevo-Type Bound for a Divergence Distance Measure. Working Paper, February.

[32] Von Neumann, J. 1955. Mathematical Foundations of Quantum Mechanics. Princeton: Princeton University Press.

[33] Wilkins, John 1707 (1641). Mercury or the Secret and Swift Messenger. London. 